\title{
Quantum Particle Swarm Optimization for Synthesis of Non-uniformly Spaced Linear Arrays with Broadband Frequency Invariant Pattern
}

\author{
Hemant Patidar, Gautam Kumar Mahanti \\ Department of Electronics and Communication Engineering, \\ National Institute of Technology, Durgapur, India \\ Hemantpatidar08@gmail.com,gautammahanti@yahoo.com \\ R Muralidharan \\ Department of Electrical and Computer Engineering, \\ Caledonian College of Engineering, \\ Sultanate of Oman \\ Email:muralidharan@caledonian.edu.om
}

\begin{abstract}
This paper describes a method using Quantum Particle Swarm Optimization to obtain a broadband frequency invariant pattern for synthesis of nonuniformly spaced linear array of isotropic antennas. Two cases related to this work using QPSO have been studied, namely, in the first case, the generated frequency invariant far-field pattern is broadside in the vertical plane and in the second case, the far-field frequency invariant pattern is scanned in a particular direction. An effort is made such that the side lobe level and first null beam widths of the patterns are made similar to their related desired values. The two cases are presented in this paper to show the effectiveness of the proposed method in achieving the desired specifications. Even though, the developed method is utilized here for a linear array of isotropic antennas; it can be extended to other type of arrays. The performance of this algorithm is validated by duly comparing it with firefly algorithm.
\end{abstract}

Index Terms - Broadband array, frequency invariant pattern, first null beam width, quantum particle swarm optimization, side lobe level, firefly algorithm.

\section{INTRODUCTION}

Broadband antenna arrays [1] are widely used in many applications such as communication, acoustics and radio systems engineering etc. because of their ability to simultaneously broadcast multiple signals and traffic types in a wide bandwidth of transmission. Frequency invariant array pattern formation is advantageous for broadband signal acquisition and is usually designed for undistorted transmission of wideband signals [2]. Many broadband pattern synthesis techniques have been utilized 
in the literature [3-6]. Array pattern synthesis techniques for designing of broadband FIR beam former with constant main lobe response and minimum side lobe level (SLL) have been discussed in [3-5]. All elements are fed coherently with a constant scan angle in phased array and phase shifters of all the elements are used to scan the beam in desired direction [6]. A technique for synthesis of narrowband and wideband array pattern using particle swarm optimization is detailed in [6].

Many methods have been proposed for the synthesis of nonuniformly spaced arrays [7-10]. Some techniques like genetic algorithm and neural- network based methodology and performance of raised power series arrays have been described in [7-8] for synthesizing wideband non-uniformly spaced arrays. The method described in [9] uses the asymptotic theory of unequally spaced arrays to produce ultra-wideband pattern with invariant main beam, but generates higher SLL. Another method for synthesis of nonuniformly spaced linear arrays with wide band frequency-invariant patterns using the generalized matrix pencil methods has been described in [10]. In this paper, a simple method is presented for broadband frequency invariant pattern using Quantum Particle Swarm Optimization (QPSO) algorithm for synthesis of nonuniformly spaced linear array antenna [11-13].

QPSO has recently enjoyed its success in the field of antenna arrays. To mention further, it was successfully employed [13] in failure correction of the radiation pattern in presence of faulty elements by newly generating the excitations. It also restored the side lobe level along with the wide null placement in the presence of faulty elements. But, in this paper, QPSO is employed in generating frequency invariant patterns and hence forth, the procedures, equations that are involved in generating these patterns are totally different from that of [13]. Moreover, these generations are tested here not only in broadside, but also in a particular direction in vertical plane for proving its versatility. In addition to it, necessary care is taken not only to control the side lobe level, but also the first null beam widths. This algorithm is used here to generate both the excitations amplitudes as well as the distance between the elements.

The reason for the choice of QPSO is because of its validity [14-16] over other algorithms. Moreover, in this paper, QPSO algorithm is compared with firefly algorithm (FA), a nature-inspired metaheuristic optimization algorithm which is described in [17-19]. Literature reports [18, 20] the superiority of FA than other basic algorithms.

\section{APPROACH FOR FREQUENCY INVARIANT PATTERN SYNTHESIS}

A non-uniformly spaced linear array of $2 N$ isotropic antennas along $Y$-axis has been considered. Elements of the array are located symmetrically on each side of the origin as shown in fig.1. The equation of broadband array factor to generate free-space far-field pattern $F I P(f, \theta)$ in the vertical plane $(Y-Z)$ with symmetric amplitude distributions is given by (1).

$$
F I P(f, \theta)=\left[\sum_{n=1}^{N} 2 I_{n} \cos \left[\frac{2 \pi f}{c} d_{n}\left(\sin (\theta)-\sin \left(\theta_{O}\right)\right)\right]\right]
$$


where $n=$ element number, $d_{n}=$ distance from origin to centre of the $n^{\text {th }}$ element, $\lambda=\mathrm{c} / f=$ radiating wavelength, $f=$ radiating frequency, $\theta$ is the polar angle of far-field measured from $Z$-axis $\left(-90^{\circ}\right.$ to $\left.+90^{\circ}\right), \theta_{0}=$ scan angle, $c=$ radiation velocity in free space, $I_{n}=$ amplitude of the excitation current at $n^{\text {th }}$ element, $N$ is the total number of elements from one side of the origin. For a broadside pattern $\theta_{0}$ will be equal to $0^{\circ}$. The range of frequency is represented by $\left[f_{L}, f_{U}\right]$. For synthesis of frequency invariant pattern with broadband, antenna array should maintain a constant main lobe (or first null beam width (FNBW)) in this frequency band.

Normalized absolute far-field in $\mathrm{dB}$ can be articulated in (2) as follows:

$$
F I P_{n}(f, \theta)=20 \log _{10}\left[\frac{|F I P(f, \theta)|}{|F I P(f, \theta)|_{\max }}\right]
$$

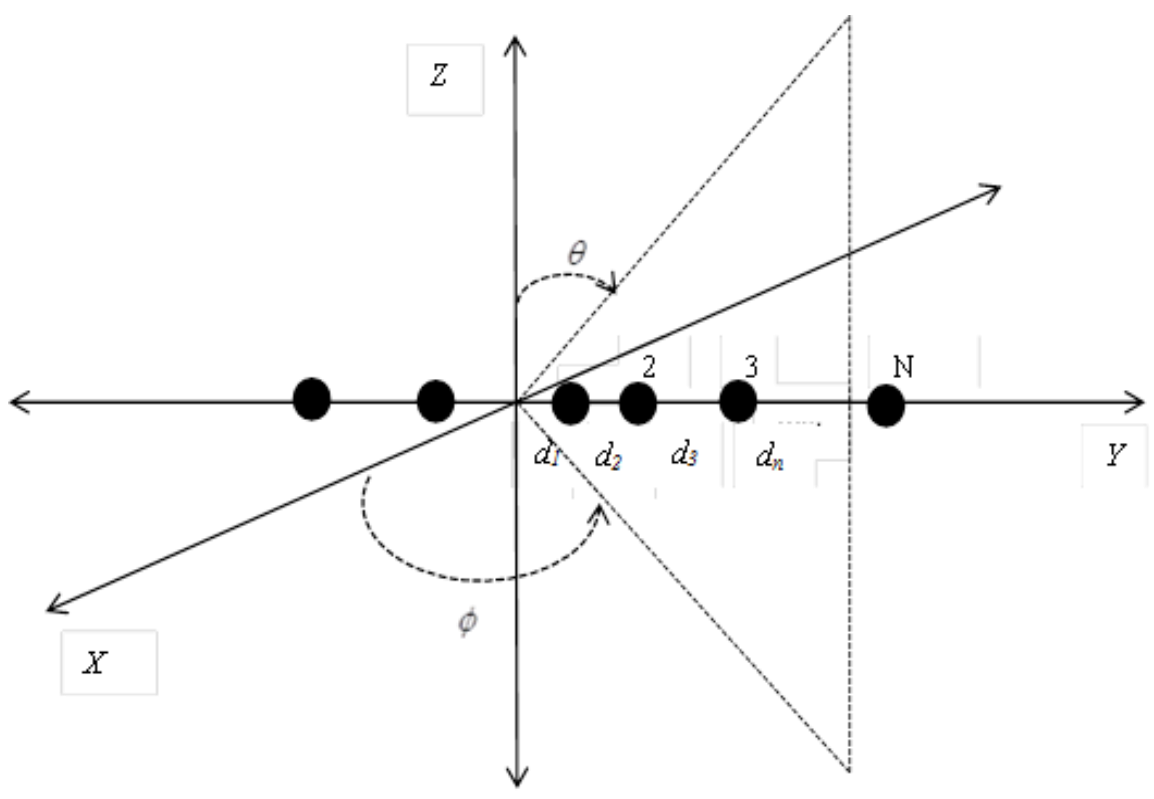

Fig. 1. Geometry of a non-uniformly spaced linear array of isotropic antennas along the $Y$-axis.

For scannable pattern in frequency band $\left[f_{L}, f_{U}\right]$, the response of array factor defined by the coefficients $I_{n}$ at any frequency is scaled by $f$ and shifted from $\theta$ to the desired angle $\theta_{0}$. But scaling by $f$ results in an array pattern that becomes narrower as the frequency increases.

The problem is to find the set of parameters $I_{n}$ and $d_{n}$ of the array elements using QPSO that will minimize the following fitness function Cost, so that minimum SLL and frequency invariant beam pattern can be achieved over frequency band range for both cases.

$$
\begin{gathered}
\text { Cost }=\left[w_{1} \times\left(\operatorname{mse}\left|\left(F_{1}\right)\right|\right)^{0.5}+w_{2} \times\left(\operatorname{mse}\left(F_{2}\right)\right)^{0.5}\right] \\
F_{1}=d_{p}-o_{p}
\end{gathered}
$$




$$
\begin{gathered}
F_{2}=\sum_{m=1}^{k} F_{3, m} \\
F_{3}=\left\{\begin{array}{l}
\left|S L L_{0}-S L L_{d}\right|, \text { if } \rightarrow S L L_{o}>S L L_{d} \\
0, \text { if } \rightarrow S L L_{o} \leq S L L_{d}
\end{array}\right.
\end{gathered}
$$

The coefficients $w_{1}$ and $w_{2}$ are the relative weights applied to each term in (3).

where $k=$ sample points, $d_{p}$ and $o_{p}$ are vector of the points for desired and obtained pattern of the main beam for all the sample points in frequency band $\left[f_{L}, f_{U}\right] . S L L_{o}$ and $S L L_{d}$ are the value of SLL of obtained and desired pattern respectively. $F_{2}$ is the summation of all the sample points in frequency range $\left[f_{L}, f_{U}\right]$ for $F_{3}$. $m s e$ is a network performance function. It measures the network's performance according to the mean of squared errors.

\section{QUANTUM PARTICLE SWARM OPTIMIZATION ALGORITHM}

A novel optimization algorithm QPSO, which is rooted on the fundamental theory of particle swarm and properties of quantum mechanics, was proposed in [11-13]. QPSO is stated only by the position vector and there is no velocity vector. The movable dealing of the particle in QPSO is different from that of the particle in standard PSO.

Several steps involved in QPSO are given below:

Step 1: Generate initial particles in the population randomly between the minimum and the maximum operating limits in the Di-dimensional space.

Step 2: Estimate the value of particle objective function.

Step 3: Current fitness value of the particle is compared with personal best (pbest) of every particle. If the current fitness value of the particle is superior, then assign the current fitness value to pbest and assign the current coordinates to pbest coordinates.

Step 4: Calculate the mean best position of all $J$ particles.

$$
\text { mbest }=\frac{1}{J} \sum_{i=1}^{J} \text { pbest }_{i}
$$

Step 5: In the entire population determine the current best fitness and its coordinates. If the current best fitness value is superior to global best (gbest), then assign the current best fitness value to gbest and assign the current coordinates to gbest coordinates.

Step 6: The vector local focus of the particle is calculated using the following equation:

$$
p_{i d}^{u}=\operatorname{rand}_{i d}^{u} *\left(\text { pbest }_{i d}\right)+\left(1-\operatorname{rand}_{i d}^{u}\right) *(\text { gbest })
$$

Step 7: Update position $\left(W_{i d}\right)$ of the $d^{\text {th }}$ dimension of the $i^{\text {th }}$ particle using the following equations: 


$$
\begin{aligned}
& W_{i d}^{u}=p_{i d}^{u}+(-1)^{\operatorname{ceil}\left(0.5+\operatorname{rand}_{i d}^{u}\right)} \times \sigma \\
& \times \mid \text { mbest }-W_{i d}^{u-1} \mid \times \log _{e}\left(1 / \operatorname{rand} 3_{i d}^{u}\right)
\end{aligned}
$$

If $\quad W_{i d}^{u}<W_{\min }^{d}$

then $\quad W_{i d}^{u}=W_{\min }^{d}+0.25 * \operatorname{rand} 4_{i d}^{u} *\left(W_{\max }^{d}-W_{\min }^{d}\right)$

If $\quad W_{i d}^{u}>W_{\max }^{d}$

then

$$
W_{i d}^{u}=W_{\max }^{d}-0.25 * \operatorname{rand} 5_{i d}^{u} *\left(W_{\max }^{d}-W_{\min }^{d}\right)
$$

where $u$ is the current generation, $r a n d 1$, rand 2 , rand 3 , rand 4 and $r a n d 5$ are uniform random numbers between 0 and 1. Equations (7) and (8) have been used along in each dimension $\left(W_{\max }^{d}, W_{\min }^{d}\right)$ to squeeze the position. These techniques are essential to stop particles from eruption if they try to cross the required domain of interest.

Step 8: Repeat Steps from 2 to 7 till the maximum number of iterations being completed or when there is no further update of best fitness value.

The parameter $p$ is the local attractor of each particle. To avoid untimely convergence mbest is considered as the barycenter of all particles. $\sigma$ is the contraction and expansion coefficient used for controlling the convergence speed and performance of the particle. This is the only one controlling parameter in QPSO, which can be tuned easily by linear variation in between minimum and maximum value or by trial and error method to control the convergence speed of the algorithm. The value of $\sigma$ has been changed for different examples with frequency invariant pattern in this paper. Setting the value of $\sigma$ in the interval $(0.5,0.8)$, can generates good results, see literature report [14].

For case 1: The value of $\sigma$ with each iteration is given by for example of 20 element linear array:

$$
\sigma=\left(\sigma_{\max }-\sigma_{\text {min }}\right) \times \frac{\left(\max _{g e n}-u\right)}{\left(\max _{g e n}-1\right)}+\sigma_{\text {min }}
$$

where $\sigma_{\max }=0.8$ and $\sigma_{\min }=0.7$ are the maximum and minimum value of $\sigma, \max _{\operatorname{gen}}$ is the maximum number of generation and $u$ is the current generation.

For example of 26 elements the value of $\sigma$ is given by:

$$
\sigma=0.71+0.1 \text { *rand; }
$$

For case 2: The value of $\sigma$ for 20 elements:

$$
\sigma=0.7+0.1 \text { *and }
$$


The value of $\sigma$ for 26 element of linear array:

$$
\sigma=0.7+0.09 * \text { rand }
$$

where rand is the uniform random numbers between 0 and 1. Flow chart of QPSO algorithm is detailed in Fig.2.

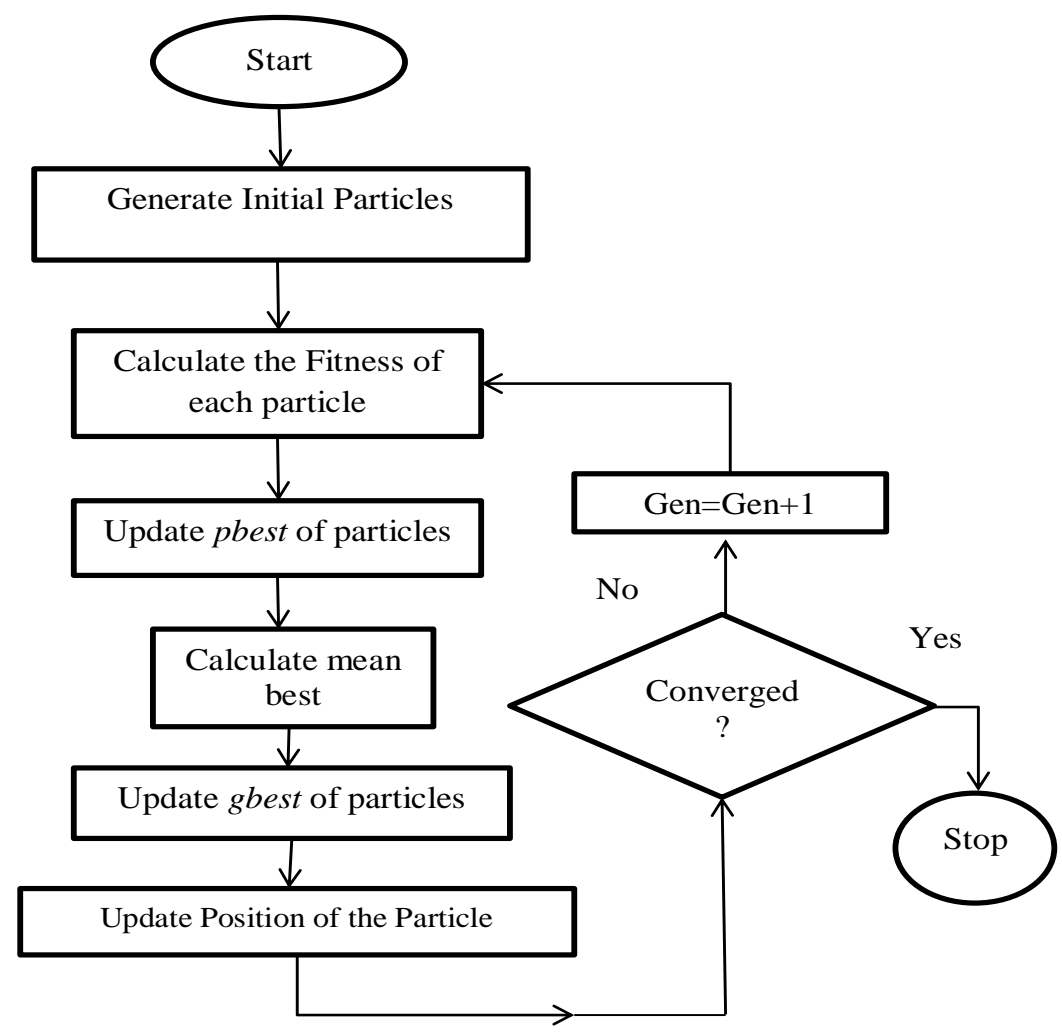

Fig. 2. Flow chart of quantum particle swarm optimization algorithm.

In addition to QPSO algorithm, Firefly Algorithm is also considered for the experiments for the sole purpose of comparisons between these two algorithms. This algorithm developed by the author XinShe Yang is based on the idealized behaviour of the attraction characteristics of fireflies [17-19]. The flashing characteristics of the fireflies are described as follows:

- Fireflies are attracted to other ones irrespective of their sex.

- The less brighter firefly will move towards the brighter one as the attractiveness is proportional to their brightness. This attractiveness is inversely proportional to the distance as it decreases as the distance between the firefly's increases. If no one is brighter than a particular firefly, it moves randomly.

- The brightness or light intensity of a firefly is affected or determined by the cost function which is to be optimized.

The settings for the firefly algorithm parameters are: Randomization parameter $=1$; Minimum value of attractiveness $=0.2$ and absorption coefficient $=1$. 


\section{SIMULATION RESULTS}

The numbers of element taken along the $Y$-axis for the simulation are 20 and 26 for both cases. Operating frequency range is $f_{L}=0.5 \mathrm{GHz}$ and $f_{U}=1 \mathrm{GHz} . \frac{f_{U}}{f_{L}}=2, \mathrm{k}=11$ (with constant increment of $0.05 \mathrm{GHz}$ ) sample points in frequency band and centre frequency is at $0.75 \mathrm{GHz}$.

Array elements are considered symmetric with respect to origin to reduce the number of variables in the simulation process. Desired SLL are fixed for both cases (20 elements and 26 elements) and it is $20 \mathrm{~dB}$. FNBW for 20 elements linear array is fixed at $13^{\circ}$ for both cases and for 26 elements linear array; it is $20^{\circ}$ for case 1 and $29^{\circ}$ for case 2 .

Two cases are discussed below.

Case 1: For generation of far-field broadside frequency invariant pattern in vertical plane QPSO and FA are run for 300 iterations with a particle size of 30. Excitation current amplitude is varied in the range 0 to 1 for both elements. The spacing between the elements is varied from $0.5 \lambda$ to $1.5 \lambda$ (for 20 element linear array) and $0.5 \lambda$ to $1.0 \lambda$ (for 26 element linear array) at the highest in-band frequency of $1 \mathrm{GHz}$ in order to suppress grating lobes at lower frequencies. All the current excitation phases are kept fixed at $0^{\circ}$. Due to symmetry, only ten excitation current amplitudes and ten distances from the origin to the centre of antenna element are to be optimized for 20 element linear array. Same thing has been done for 26 element linear array also. Algorithms has been utilized to generate a vector of 20 and 26 real values, first 10 and 13 values for current amplitude and last ten and thirteen values are for distance of antenna element from origin.

Case 2: For generation of scanned far-field frequency invariant pattern in principal vertical plane (YZplane) QPSO and FA are run for 300 iterations with a particle size of 30. Excitation current amplitude is varied in the range 0 to 1 for 20 and 26 elements. The spacing between the elements is varied from $0.4 \lambda$ to $0.7 \lambda$ (for 20 element linear array) and $0.3 \lambda$ to $0.9 \lambda$ (for 26 element linear array) at the highest in-band frequency of $1 \mathrm{GHz}$ in order to suppress grating lobes at lower frequencies. All the current excitation phases are kept fixed at $25^{\circ}$ (for 20 element linear array) and $45^{\circ}$ (for 26 element linear array) for scanned far-field pattern.

Matlab software has been used here. Computational time is measured here using a PC with Intel(R) Core(TM) i5-4690 processor of clock frequency $3.50 \mathrm{GHz}$ and $4 \mathrm{~GB}$ of RAM. Measured computational time for case 1 (20 element and 26 element for QPSO) are 633.68 seconds and 703.69 seconds and case 2 (20 element and 26 element for QPSO) are 665.43 seconds and 720.12 seconds. For firefly algorithm the computational time for case 1 are 638.16 and 667.72 and case 2 are 704.52 and 722.42 seconds. 
Table I and II shows the current amplitude distribution and distance of antenna elements from origin for 20 elements and 26 elements. Fig. 3 and Fig. 4 shows the global best fitness value versus iterations obtained for both cases for 20 elements and 26 elements linear array. Fig. 5 and Fig. 6 shows the normalized current amplitude distribution using QPSO and FA for 20 and 26 elements. Fig. 7 shows the normalized frequency invariant power pattern in $\mathrm{dB}$ for 20 element linear arrays obtained from QPSO and FA for case 1. Fig. 8 shows the scanned normalized frequency invariant power pattern in $\mathrm{dB}$ for 20 element linear arrays obtained from QPSO and FA. Fig. 9 shows the normalized frequency invariant power pattern in $\mathrm{dB}$ for 26 element linear arrays using QPSO and FA for case 1. Fig. 10 shows the scanned normalized frequency invariant power pattern in $\mathrm{dB}$ for 26 element linear arrays using QPSO and FA.

It is observed from Fig. 3 and Fig. 4 that the algorithms reached to a value after which it is not converged after 200 iterations. QPSO converged well as compared to FA for both the cases. From the obtained pattern in Fig. 7 and Fig. 9, it is observed that error in obtained frequency invariant pattern and in desired pattern is less using QPSO and FA means constant frequency invariant pattern are obtained using both the algorithms; whereas in Fig. 8 the pattern obtained using FA provides higher side lobe level as compared to obtained pattern using QPSO algorithm. Moreover, in Fig.10 pattern obtained using FA provides more error in desired and obtained main beam patterns than QPSO.

TABLE I. EXCITATION CURRENT AMPLITUDE AND SPACING FROM ORIGIN

\begin{tabular}{|c|c|c|c|c|c|c|c|c|}
\hline \multirow{2}{*}{ Element No } & \multicolumn{4}{|c|}{ For Case 1 (20 Element) } & \multicolumn{3}{c|}{ For Case 2 (20 Element) } \\
\cline { 2 - 9 } & Current Excitation & \multicolumn{2}{|c|}{ Spacing $($ in $\lambda$ ) } & Current Excitation & \multicolumn{2}{c|}{ Spacing( in $\lambda$ ) } \\
\cline { 2 - 9 } & QPSO & FA & QPSO & FA & QPSO & FA & QPSO & FA \\
\hline \pm 1 & 0.7128 & 0.8399 & 0.5060 & 0.5144 & 0.8475 & 0.8614 & 0.4200 & 0.4163 \\
\hline \pm 2 & 0.6067 & 0.9660 & 1.3514 & 1.4400 & 0.8319 & 0.9433 & 1.0089 & 0.9908 \\
\hline \pm 3 & 0.6473 & 0.8757 & 2.3260 & 2.3213 & 0.7244 & 0.6933 & 1.6532 & 1.6674 \\
\hline \pm 4 & 0.7282 & 0.6932 & 3.2446 & 3.2206 & 0.7975 & 0.8399 & 2.3492 & 2.2979 \\
\hline \pm 5 & 0.5867 & 0.4572 & 4.1545 & 4.2298 & 0.6036 & 0.6090 & 2.9940 & 2.9420 \\
\hline \pm 6 & 0.4717 & 0.4214 & 5.1110 & 5.1686 & 0.6606 & 0.7243 & 3.6853 & 3.6047 \\
\hline \pm 7 & 0.4254 & 0.5080 & 6.0553 & 6.1739 & 0.4740 & 0.4557 & 4.3113 & 4.2213 \\
\hline \pm 8 & 0.4308 & 0.4409 & 6.9527 & 7.1188 & 0.5150 & 0.5490 & 5.0110 & 4.8857 \\
\hline \pm 9 & 0.3216 & 0.1896 & 7.8158 & 8.0643 & 0.5374 & 0.6388 & 5.6972 & 5.5022 \\
\hline \pm 10 & 0.3663 & 0.4100 & 8.7086 & 9.0013 & 0.5754 & 0.6370 & 6.3179 & 6.0607 \\
\hline
\end{tabular}


TABLE II. EXCITATION CURRENT AMPLITUDE AND SPACING FROM ORIGIN

\begin{tabular}{|c|c|c|c|c|c|c|c|c|}
\hline \multirow{2}{*}{ Element No } & \multicolumn{4}{|c|}{ For Case 1 (26 Element) } & \multicolumn{4}{c|}{ For Case 2 (26 Element) } \\
\cline { 2 - 9 } & Current Excitation & \multicolumn{2}{|c|}{ Spacing( in $\lambda$ ) } & Current Excitation & \multicolumn{3}{c|}{ Spacing( in $\lambda$ ) } \\
\cline { 2 - 9 } & QPSO & FA & QPSO & FA & QPSO & FA & QPSO & FA \\
\hline \pm 1 & 0.8967 & 0.8571 & 0.5104 & 0.5115 & 0.6849 & 0.8607 & 0.3044 & 0.3045 \\
\hline \pm 2 & 0.9840 & 0.8671 & 1.2115 & 1.2744 & 0.7998 & 0.7709 & 0.8254 & 0.8256 \\
\hline \pm 3 & 0.7872 & 0.8631 & 1.8956 & 2.0477 & 0.6788 & 0.7378 & 1.3783 & 1.3888 \\
\hline \pm 4 & 0.7356 & 0.6790 & 2.5033 & 2.8339 & 0.6501 & 0.7111 & 1.9676 & 1.9542 \\
\hline \pm 5 & 0.6669 & 0.6669 & 3.1487 & 3.6280 & 0.5558 & 0.7127 & 2.4909 & 2.4472 \\
\hline \pm 6 & 0.6026 & 0.5710 & 3.8741 & 4.3514 & 0.5624 & 0.4789 & 3.0282 & 2.9982 \\
\hline \pm 7 & 0.4578 & 0.4677 & 4.4639 & 5.2408 & 0.4421 & 0.3588 & 3.6014 & 3.6629 \\
\hline \pm 8 & 0.3970 & 0.4908 & 5.1461 & 5.9887 & 0.4956 & 0.5281 & 4.1784 & 4.1673 \\
\hline \pm 9 & 0.3322 & 0.2359 & 5.9623 & 6.8003 & 0.4103 & 0.3435 & 4.7779 & 4.6534 \\
\hline \pm 10 & 0.0966 & 0.0000 & 6.7450 & 7.6297 & 0.5274 & 0.4144 & 5.2423 & 5.2569 \\
\hline \pm 11 & 0.6959 & 0.6011 & 7.6696 & 8.3301 & 0.3749 & 0.3566 & 6.0434 & 5.9064 \\
\hline \pm 12 & 0.8593 & 0.4861 & 8.3086 & 9.1841 & 0.0432 & 0.4064 & 6.4410 & 6.4595 \\
\hline \pm 13 & 0.9605 & 0.1728 & 8.8394 & 9.9364 & 0.0810 & 0.6314 & 7.0765 & 6.8373 \\
\hline
\end{tabular}

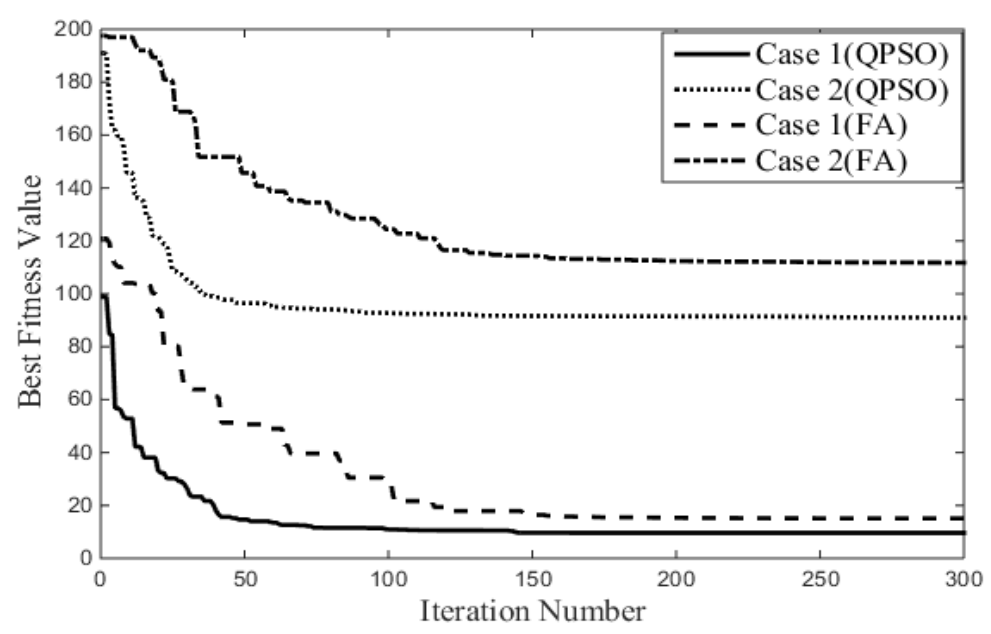

Fig. 3. Global best fitness value versus iteration number obtained from QPSO and FA for 20 element linear array.

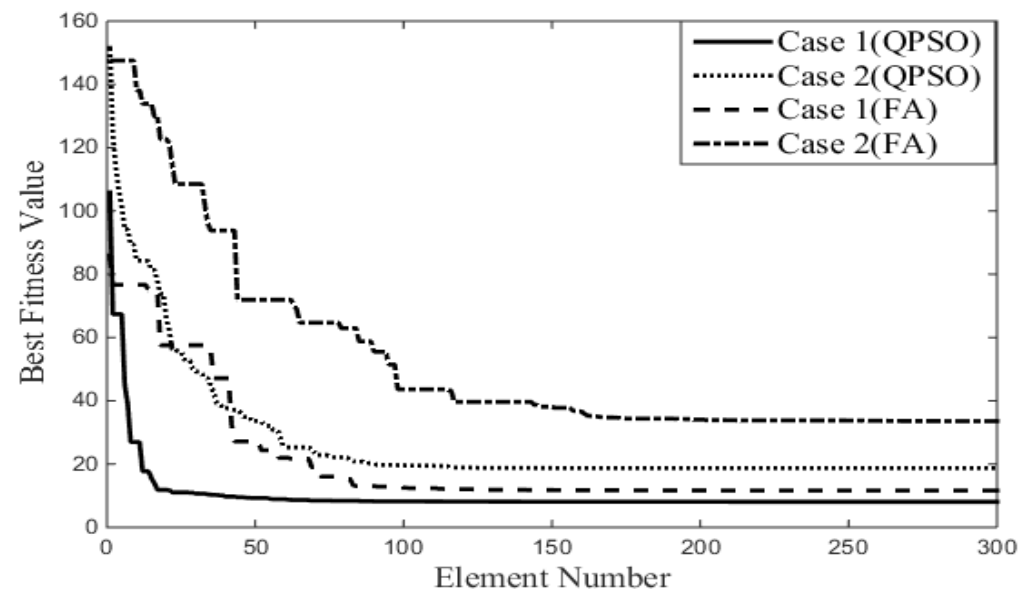

Fig. 4. Global best fitness value versus iteration number obtained from QPSO and FA for 26 element linear array. 


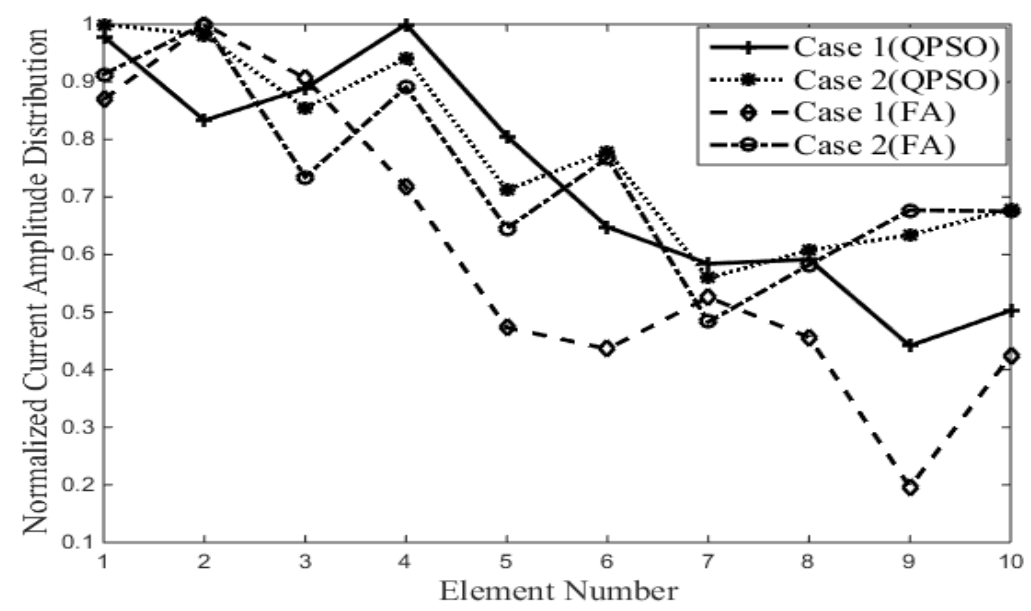

Fig. 5. Normalized current amplitude distribution for one side of the element from origin using QPSO and FA for 20 element linear array.

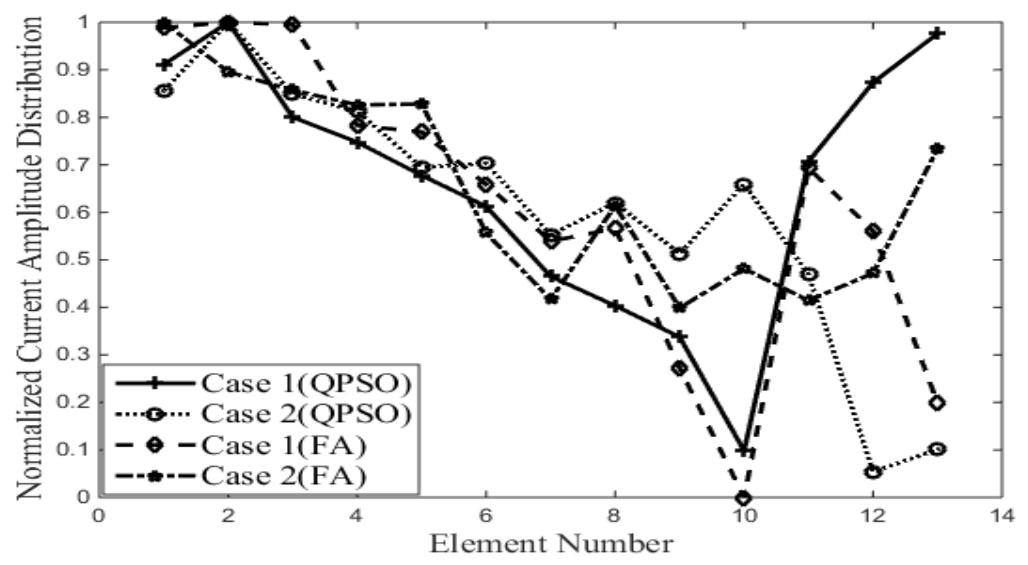

Fig. 6. Normalized current amplitude distribution for one side of the element from origin using QPSO and FA for 26 element linear array.

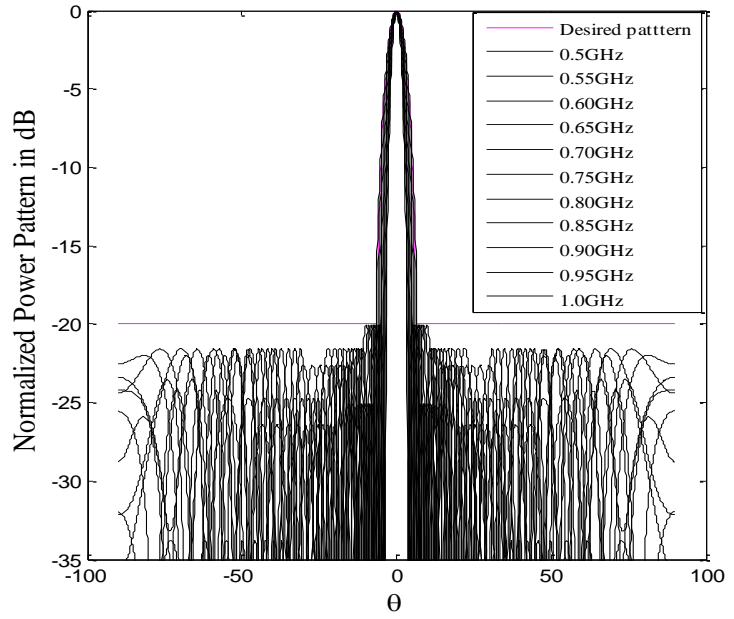

(a) Normalized Power Pattern in dB using QPSO

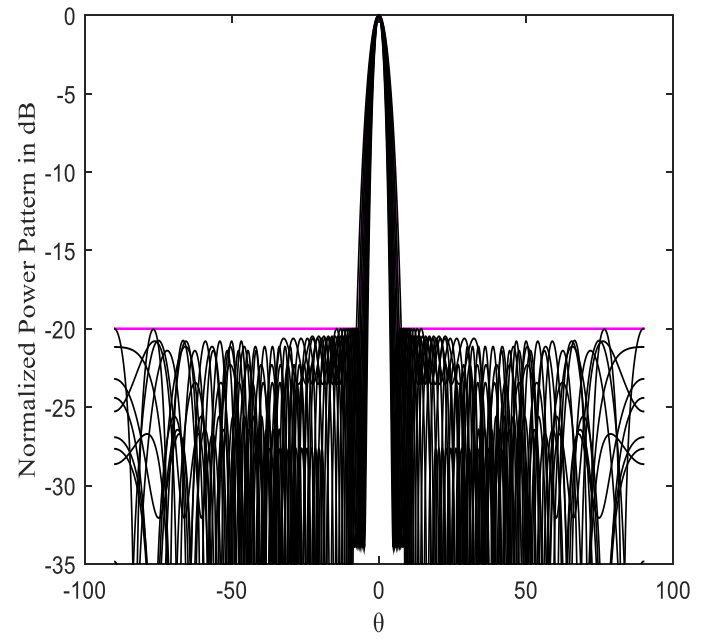

(b) Normalized Power Pattern in dB using FA

Fig. 7. Normalized Frequency invariant far-field pattern for synthesis of nonuniformly spaced broadband linear array antenna using QPSO and FA (case 1) for 20 element. 


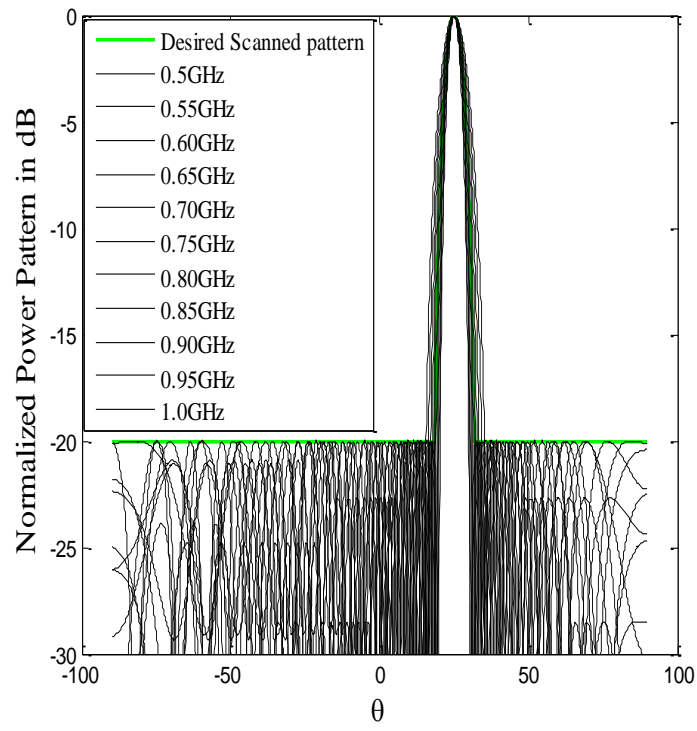

(a) Normalized Power Pattern in $\mathrm{dB}$ using QPSO

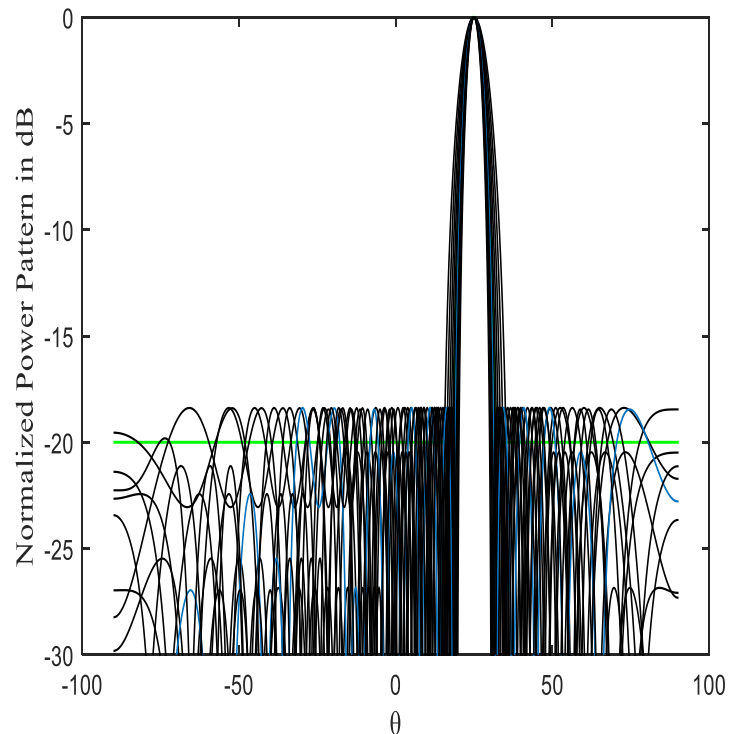

(b) Normalized Power Pattern in dB using FA

Fig. 8. Normalized Frequency invariant far-field pattern for synthesis of nonuniformly spaced broadband linear array antenna using QPSO and FA (case 2) for 20 element.

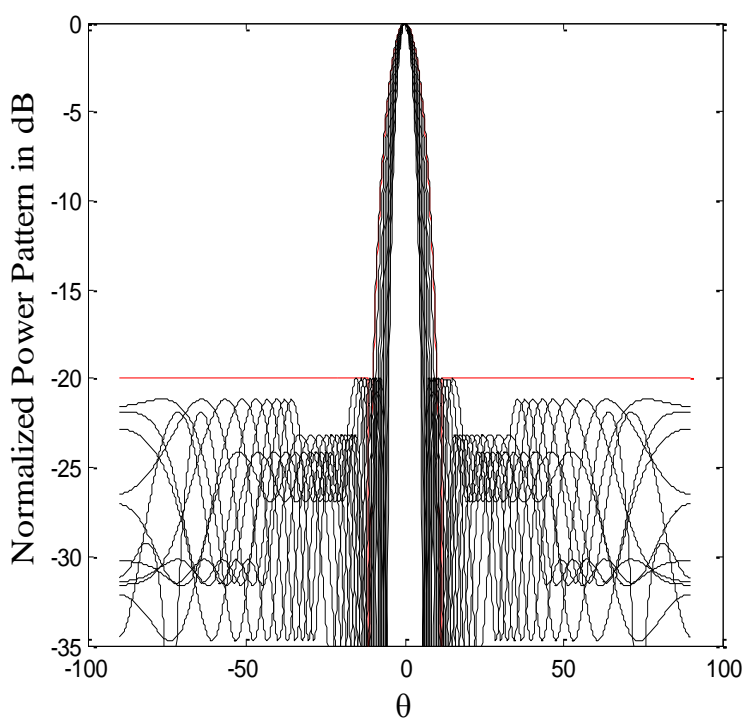

(a) Normalized Power Pattern in dB using QPSO

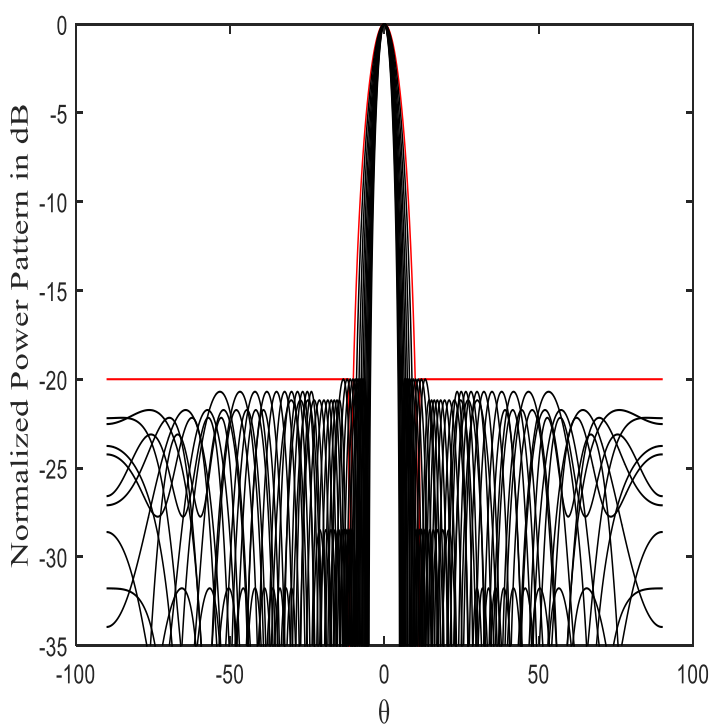

(b) Normalized Power Pattern in dB using FA

Fig. 9. Normalized Frequency invariant far-field pattern for synthesis of nonuniformly spaced broadband linear array antenna using QPSO and FA (case 1) for 26 element. 


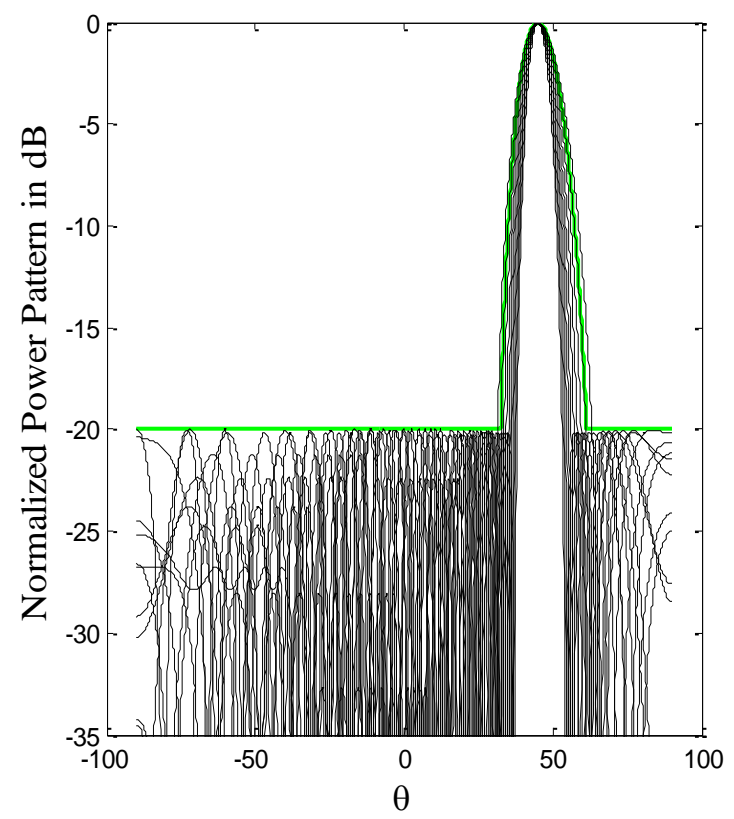

(a) Normalized Power Pattern in dB using QPSO

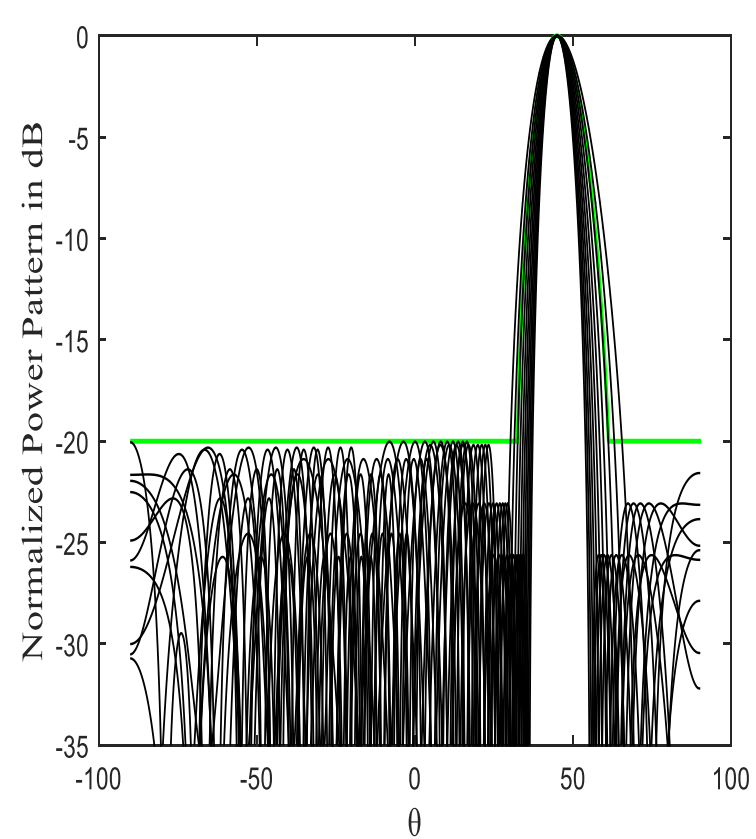

(b) Normalized Power Pattern in dB using FA

Fig. 10. Normalized Frequency invariant far-field pattern for synthesis of nonuniformly spaced broadband linear array antenna using QPSO and FA (case 2) for 26 element.

\section{CONCLUSION}

In this paper a method based on quantum particle swarm optimization algorithm has been successfully used for synthesis of a non-uniformly spaced linear array for broadband frequency invariant far-field pattern with fixed SLL and different constant FNBW (main beam). Simulated results obtained clearly show a great deal between the desired and obtained specifications. This technique works well for different width of main beam with different antennas for both cases. This technique is suitable to maintain constant SLL and nearly constant FNBW for both cases as SLL and FNBW are contradictory to each other. Algorithm finds suitable excitation current amplitudes and spacing between the elements to obtain the desired values. From the obtained results of the above figures it is proved that QPSO algorithm is more suitable for broadband frequency invariant pattern of linear antenna array. This work can be extended to other type of arrays. This proposed technique is suitable to generate frequency invariant pattern with different main beam shapes also.

\section{REFERENCES}

[1] C. A. Balanis, "Antenna theory: analysis and design," 2nd ed. Singapore: John Wiley and Sons, Asia, 2003.

[2] D. B. Ward et al., "Theory and design of broadband sensor arrays with frequency invariant far-field beam patterns," $J$. Acoust. Soc. Amer., vol. 97, pp.1023-1034, February 1995.

[3] S. Yan and C. Hou, "Broadband DOA estimation using optimal array pattern synthesis technique," IEEE Antennas Wireless Propag. Lett, vol. 5, no.1, pp. 88-90, 2006.

[4] S. Yan et al., "Optimal array pattern synthesis for broadband arrays," J. Acoust. Soc. Amer., vol. 122, no. 5, pp. 2686- 
2696, November 2007.

[5] N. Xie et al., "Broadband frequency invariant beamformer," Wireless Pers. Commun., vol. 61, no.1, pp. 143-159, November 2011.

[6] H. M. Elkamchouchi and M. M Wagih, "Synthesis of wideband array patterns via particle swarm optimization," in Proc. ITI 5th Int. Conf. Information and Communications Technology, 2007, pp.109-113.

[7] C. S. Deluccia and D. H Werner, "Nature-based design of aperiodic linear arrays with broadband elements using a combination of rapid neural network estimation techniques and genetic algorithms," IEEE Antennas and Propag., vol. 49, no.5, pp.13-23, October 2007.

[8] M. D. Gregory and D. H. Werner, "Ultra wideband aperiodic antenna arrays based on optimized raised power series representations," IEEE Trans. Antennas Propag., vol. 58, no. 3, pp. 756-764, March 2010.

[9] J. H. Doles and F. D. Benedict, "Broad-band array design using the asymptotic theory of unequally spaced arrays," IEEE Trans. Antennas Propag., vol. 36, no.1, pp. 27-33, January 1988.

[10] Y. Liu et al., "Synthesis of nonuniformly spaced linear arrays with frequency-invariant patterns by the generalized matrix pencil methods, "IEEE Trans. Antennas Propag., vol. 63, no. 4, pp.1614-1625, April 2015.

[11] J. Sun et al., "Particle swarm optimization with particles having quantum behavior," in: Proc. of congress on evolutionary computation, USA, 2004, pp. 325-331.

[12] S. M. Mikki and A. A. Kishk, "Quantum particle swarm optimization for Electromagnetics," IEEE Transactions on Antennas and Propagation. vol. 54, no.10, pp. 2764-2775, October 2006.

[13] R. Muralidaran et al., "QPSO for failure correction of linear array antenna including wide null placement," ICTACT Journal on Communication Technology, vol. 5, no.3, pp. 959-962, September 2014.

[14] J. Sun et al., "Quantum behaved particle swarm optimization with Gaussian distributed local attractor point," J. Appl. Math. Comput., vol. 218, pp. 3763-3775, 2011.

[15] J. sun et al., "Quantum-behaved particle swarm optimization: analysis of individual particle behavior and parameter selection," Evolutionary Computation, vol. 20, no.3, pp. 349-393, 2012.

[16] R. Muralidaran et al., "QPSO versus BSA for failure correction of linear array of mutually coupled parallel dipole antennas with fixed side lobe level and VSWR," Advances in Electrical Engineering, vol. 2014, Article ID 858290, pp. $1-7,2014$.

[17] X. S. Yang, "Firefly algorithm, stochastic test functions and design optimization," International Journal of BioInspired Computation, vol.2, no.2, pp.78.84, 2010.

[18] X.S. Yang, "Firefly Algorithm, L'evy Flights and Global Optimization," Research and Development in Intelligent Systems, pp. 209.218, 2010.

[19] R. Muralidharan et al., "Firefly algorithm for failure correction of linear array of dipole antennas in presence of ground plane with mutual coupling effects,” ACES journal, vol. 30, no. 10, pp. 1122-1128, 2015.

[20] G. Ram et al., "Optimized hyper beamforming of receiving linear antenna arrays using Firefly algorithm," International Journal of Microwave and Wireless Technologies, vol.6, no.2, pp-181-194, 2014. 